\title{
Power-law autocorrelation of neural activity in models of mental states that are hierarchically organized Fabian Chersi*1, Mattia Rigotti ${ }^{2}$ and Stefano Fusi ${ }^{1,2}$
}

\author{
Address: ${ }^{1}$ Institute of Neuroinformatics, University and ETH Zurich, Zurich, Switzerland and ${ }^{2}$ Center for Theoretical Neuroscience, Columbia, New \\ York, USA \\ Email: Fabian Chersi* - chersi@rocketmail.com \\ * Corresponding author
}

from Eighteenth Annual Computational Neuroscience Meeting: CNS*2009

Berlin, Germany. 18-23 July 2009

Published: 13 July 2009

BMC Neuroscience 2009, I0(Suppl I):P292 doi:I0.I |86/I47|-2202-I0-SI-P292

This abstract is available from: http://www.biomedcentral.com//47/-2202/I0/SI/P292

(c) 2009 Chersi et al; licensee BioMed Central Ltd.

We assume that complex tasks are executed by going through a series of inner mental states, each representing an actively maintained disposition to behavior. Each state contains task relevant information about past events and internal cognitive processes. For example, in complex tasks the same sensory stimulus might require different motor responses depending on the context. More generally, decisions are affected by a large number of factors such as motivations, emotions and intentions, which are all assumed to be encoded in the inner mental state. Every task relevant event induces a transition from one mental state to another. This implies that mental states are numerous and the event driven transitions between them are highly organized.

We constructed tasks in which the schemes of mental states and event driven transitions are hierarchically organized and scalable in complexity. We then built a model of a neural network that performs these tasks. We assumed that mental states are represented by stable attractors of neural dynamics, with external events steering the activity from one state to another. The structure of mental states and event driven transitions is encoded in the synaptic couplings between neurons, and it contains the instructions to react to the events occurring in the external world.

We show that there is a wide class of hierarchically organized schemes of mental states and event driven transitions that produce neural activity with long-range temporal cor- relations. In particular, the autocorrelation function of the neural activity decays as a power-law on a time interval that increases linearly with the number of mental states. The power-law behavior is entirely due to the hierarchical structure of the scheme of mental states as the events are always assumed to be delta correlated. Power-law autocorrelations are widely observed in recorded activity in behaving animals [1].

Highly variable neural activity might also be an expression of the dynamics of a neural network implementing a complex scheme of mental states and event driven transitions. Experiments show that there are rapid changes in the recorded neural activity that can be interpreted as sudden transitions from one state to another $[2,3]$. These transitions can occur also in the interval between two task relevant events, indicating that there might be multiple processing stages during the perception of a sensory stimulus. Such stages would correspond to different mental states and the transitions would be generated either internally, by other cognitive processes, or externally, by events that are not under control in the experimental protocol. The fluctuations in the neural activity caused by these frequent transitions have a $1 / f$ power spectrum in our model. If these fluctuations are strong enough to affect the final motor response or our perceptual decisions, then our model can also explain the $1 / \mathrm{f}$ noise widely observed in human cognition and performance [4]. 


\section{Acknowledgements}

We would like to thank the Swiss National Science Foundation for the support contributed through the SNF grant PPO0A-I06556.

\section{References}

I. Leopold DA, Murayama Y, Logothetis N: Very slow activity fluctuations in monkey visual cortex: implications for functional brain imaging. Cereb Cortex 2003, 13:422-433.

2. Abeles M, Bergman H, Gat I, Meilijson I, Seidemann E, Tishby N, Vaadia E: Cortical activity flips among quasi-stationary states. Proc Natl Acad Sci USA 1995, 92:8616-8620.

3. Jones ML, Fontanini A, Sadacca BF, Miller P, Katz DB: Natural stimuli evoke dynamic sequences of states in sensory cortical ensembles. Proc Natl Acad Sci USA 2007, 104: 18772-18777.

4. Gilden DL, Thornton T, Mallon MW: I/f noise in human cognition. Science 1995, 267:1837-1839.

Publish with Biomed Central and every scientist can read your work free of charge

"BioMed Central will be the most significant development for disseminating the results of biomedical research in our lifetime. "

Sir Paul Nurse, Cancer Research UK

Your research papers will be:

- available free of charge to the entire biomedical community

- peer reviewed and published immediately upon acceptance

- cited in PubMed and archived on PubMed Central

- yours - you keep the copyright

Submit your manuscript here:

http://www.biomedcentral.com/info/publishing_adv.asp 